\title{
La lectura como práctica sociocultural y herramienta para lograr la equidad social a partir de la enseñanza
}

\author{
Discusión acerca la enseñanza de la lectura
}

\author{
Reading as a socio-cultural practice and as a tool \\ to achieve equity in education
}

«Estas buenas gentes -añadió Goethe- no saben el esfuerzo y el tiempo que cuesta aprender a leer ¡Hace ochenta años que me dedico a ello y, en verdad, no puede decirse que haya alcanzado aún la meta!» (Johann Peter Eckermann, «Conversaciones con Goethe»)

\section{Edith Silveira Caorsi*}

\section{Resumen}

Este trabajo surge de la reflexión acerca de la enseñanza de la lectura en el sistema educativo uruguayo a partir de mi participación en el equipo de ProLEE de Codicen en el año 2011. Es parte de los desafíos y las preguntas que se nos plantearon como equipo. En él aparecen cuestiones referidas a la enseñanza de la lectura como actividad institucional. En esa búsqueda y en esas discusiones decantaron las ideas que aquí expongo para mejorar la enseñanza y a potenciar los aprendizajes cargándolos de significación.

Parto de la base de que el aprendizaje de la lectura es el fundamento de la enseñanza académica ya que la alfabetización es la llave de entrada a la cultura institucional. Me pregunto acerca de los modos y las prácticas de la enseñanza de la lectura.

En la escuela, como agente universal del acceso a la cultura escrita, se realizan prácticas pedagógicas y existen representaciones sociales sobre el asunto. La enseñanza de la lectura es una instancia cruzada por tensiones históricas, sociales y políticas.

El trabajo se propone polemizar acerca de las prácticas actuales y se pregunta sobre la necesidad de una nueva narrativa que construya una epistemología y una metodología acordes a una acción basada en aspectos socioculturales con el aporte de otras ciencias sociales. Invita a desarrollar la investigación cualitativa en relación con el estado y a revisar las categorías establecidas. El objetivo de esta búsqueda es lograr una acción más eficaz en los diferentes contextos pedagógicos y potenciar la adquisición de la llamada literacidad crítica la autonomía del lector en diferentes contextos pedagógicos.

Es decir, situar la enseñanza de la lectura como una práctica potente para los docentes y significativa para todos los aprendices.

PALABRAS CLAVE: Enseñanza, lectura, sistema educativo, alfabetización 


\section{Abstract}

This work arises from reflection on the teaching of reading in the Uruguayan educational system, from my participation in the ProLEE team of Codicen in 2011. It is part of the challenges and questions we encountered as a team. In it there are issues relating to the teaching of reading as an institutional activity. From that search and those discussions decanted the ideas I here expose, not as conclusions or closures to that experience but as thresholds to cross and possible actions that lead to improve the teaching and enhance the learning by loading them with meaning.

I assume that learning to read is the foundation of academic instruction, and that literacy is the key to entering into institutional culture.

At school, as a universal agent of access to written culture, pedagogical practices are carried out and there are social representations on the matter. The teaching of reading is an instance crossed by historical, social and political tensions.

The paper aims at discussing about current practices and questions the need for a new narrative to build an epistemology and a methodology according to an action based on sociocultural aspects with input from other social sciences.

It invites to develop qualitative research regarding the state of the art and to review established categories.

The aim of this search is to achieve a more effective action in different educational contexts and enhance the acquisition of the so-called "critical literacy" "the autonomy of the reader" in different educational contexts.

That is, to establish the teaching of reading as a powerful practice for teachers and a meaningful one for all students.

KEY WORDS: teaching, reading, educational system, literacy

\section{Objetivo}

Intentaré realizar una síntesis de las posiciones y tratamiento que se le han dado al tema y exponer una parte del estado en cuanto a ello, con la finalidad de demostrar que la enseñanza de la lectura crítica desde una mirada sociocultural (lo que en el ámbito anglosajón se denomina como «literacy») podría producir un cambio en la calidad de la educación.

Creo que es hora de revisar nuestras prácticas de enseñanza y de evaluación para alcanzar la meta de construir lectores.

\section{Situación del tema}

Luego de las reformas instaladas por las Revoluciones Americana y Francesa, el proyecto de la modernidad cuyos postulados se realizan a partir de una nueva concepción de la sociedad y del ciudadano considera la alfabetización universal como un postulado fundamental, en estrecha relación con la concepción de la igualdad de los seres humanos. «Todos los problemas de la alfabetización comenzaron cuando se decidió que escribir no era una profesión sino una obligación y que leer no era marca de sabiduría sino marca de ciudadanía». Ferreiro (2000).

Desde una nueva concepción en las metas de educación de los diferentes países, el grado de alfabetización ha integrado tanto las aspiraciones como las prácticas educativas y se ha vuelto uno de los aspectos que definen el progreso de las sociedades.

En épocas anteriores tanto la enseñanza universal de la lectura como su práctica se realizaban en textos escritos en papel, libros y publicaciones periódicas, los géneros tenían límites claros y definidos y la recepción de la lectura como práctica tenía reglas accesibles para todos los que manejaban el código de la escritura en una lengua. 
El asunto de la alfabetización conserva estas referencias originales pero, si lo vemos desde las prácticas educativas de hoy, el tema de la enseñanza de la lectura se ha complejizado. Esto ocurre porque el hecho de leer también se ha vuelto complejo y múltiple. Han aparecido nuevos soportes que proponen otras instancias para la lectura y los límites de los géneros se han desdibujado, la lectura y lo legible tienen múltiples posibilidades y se ha vuelto una actividad demandante y compleja, más allá de la capacidad instalada en la mayoría de los lectores.

Ya situados en el asunto de su enseñanza, observamos que la lectura es una actividad básica y transversal a todas las demás acciones de enseñar y es por medio de ella que se evalúan todos los aprendizajes, por lo tanto su manejo con destreza es crucial para la adquisición del conocimiento, para la acreditación y para la actividad social y ciudadana.

Dice Cassany: "leer y escribir no son solo tareas lingüísticas o procesos psicológicos, sino también prácticas socioculturales. Leer exige descodificar la prosa y recuperar los implícitos, pero también darse cuenta del significado que cada comunidad otorga a una palabra" (Tras las líneas, 2006:10). Estos aspectos socioculturales y políticos de la lectura han resultado invisibles e implícitos en el tratamiento pedagógico. Esto ha incidido en la ajenidad de la práctica de la lectura para muchos de los aprendices.

\section{Los problemas}

Los fracasos relativos y absolutos en la adquisición de la capacidad de leer demuestran que la enseñanza hoy no está provocando aprendizajes significativos. En la mejor circunstancia la alfabetización conlleva un fracaso relativo, los sujetos permanecen en el sistema y pasan de grado, pero no demuestran una capacidad crítica en sus lecturas, y su comprensión de la información e intención de los textos es limitada. Estos alumnos están en la categoría de los iletrados propuesta por Anne Marie Chartier y Jean Hébrard (2002) a partir de su indagación de los discursos sociales sobre la lectura en Francia.

En el peor de los casos el fracaso es absoluto y dentro de una institución letrada como es la escuela el sujeto se frustra y abandona los cursos, lo que produce una cierta cantidad de jóvenes excluidos y desesperanzados en cuanto a su futuro.

Tal como se expresó antes, la lectura como actividad es básica y transversal a todas las actividades de enseñanza y por medio de ella se evalúan todos los aprendizajes, por lo tanto es crucial para el desarrollo del conocimiento y por último, pero no menos importante, es crucial para desenvolverse con plenitud en la actividad ciudadana. La hegemonía del discurso psicolingüístico en la pedagogía de la lectura ha centrado el tema en la categoría comprensión lectora y en los problemas referidos al uso social del lenguaje (diferentes variantes de la oralidad, por ejemplo). Esto ha resultado en la ajenidad de la práctica de la lectura para muchos de los aprendices.

Llegar a la lectura que va más allá de la decodificación del texto -a la alfabetización crítica con nuestros estudiantes- es un problema a solucionar.

Existen dificultades en las prácticas de lectura en todos los niveles institucionales en grados diversos y con consecuencias diferentes.

En los dos primeros niveles educativos, primaria y secundaria, es uno de los factores determinantes en la deserción escolar.

En el ámbito de la educación terciaria es asunto de límites en el aprendizaje. Esto ha sido constatado por las pruebas normalizadas en algunas facultades de la UDELAR y por las dificultades consignadas por los docentes de toda la educación terciaria. 
Vemos, por lo tanto, que el asunto es abordado desde muy diferentes ámbitos y evaluado desde distintos puntos de vista. En las representaciones sociales -el discurso de la opiniónaparecen ideas fuerza que se repiten: «ahora se lee menos; la tecnología atenta contra la capacidad y el hábito de leer; la lectura como actividad tiende a desaparecer; etc.».

Desde el lado de la epistemología el asunto es complejo. En los debates de políticas educativas estatales la concepción que subyace, aunque no de forma explícita, es que:

Leer es una actividad básica del conocimiento, que se desarrolla de una manera unívoca y que todos los alfabetizados poseen por igual. Esta concepción conduce por un camino de pensamiento que vuelve confusas las acciones de enseñanza. La confusión parte de la inmovilidad de los objetivos de la enseñanza de la lectura y esta concepción confunde los aprendizajes. Para dilucidar estos problemas considero necesario:

Definir el concepto de lectura y la tipología de la misma

Indicar la situación y el lugar de la lectura en la enseñanza institucional y en las representaciones sociales. Describir y ejemplificar la literacidad crítica y sus posibles prácticas pedagógicas

\section{La lectura}

Desde las definiciones presentes en los diccionarios hasta la reflexión de la investigación no existe hoy un consenso acerca de la concepción de la lectura, pero sí existe una coincidencia en cuanto a la polisemia de los términos que la designan. Por otro lado y como aporte de la semiótica se ha ampliado esa noción más allá del campo de los textos escritos a un corpus mayor que incluye otros aspectos como el movimiento, el sonido, lo iconográfico, los discursos orales.

\section{Algunas definiciones acerca de la lectura desde el enfoque sociocultural}

En el programa de fomento a la lectura y la escritura del CODICEN (ProLEE, 2011) consideramos que: « la lectura [es] una práctica social y cultural [ ] Estamos convencidos de que la lectura y la escritura no pueden considerarse solamente un problema pedagógico o como una acción individual, sino ante todo como una práctica profundamente socializada».

Mendoza Fillola (1991:316-319) sostiene que: « la lectura es una compleja actividad del conocimiento, en la que intervienen el dominio global de destrezas y habilidades lingüísticas, el dominio de la pragmática comunicativa, los conocimientos enciclopédicos, lingüísticos, paralingüísticos, intratextuales e intertextuales y la misma experiencia extra lingüística que posea el lector».

La lectura es una actividad de interpretación que requiere de la capacidad de asociación y creación entre la información que ya se tiene y la información que el texto nos proporciona. Entender la lectura como una práctica sociocultural implica cuestionarse sobre su funcionalidad, considerar que se relaciona estrechamente con la construcción de la imagen social de cada sujeto. Desde lo sociocultural la lectura se define como una "práctica» porque es una acción con intención, cargada de valores variables de acuerdo al contexto. Debido a la posibilidad de distintas lecturas de un mismo texto resulta que en esta acción de leer coexisten diferentes modos de re-producción y de interpretación.

Puestos estos postulados en la perspectiva de un análisis científico histórico, Álvarez Zapata (2002:138) propone una categoría muy potente a la que llama «modo de producción y consumo textual (MOCT)». Sostiene que: «...cuando se califica a la lectura como práctica sociocultural, se está haciendo alusión a un conjunto de hechos y situaciones en ella y por 
ella suscitado, relacionado con los efectos socializantes y simbólicos (funciones, finalidades, valoraciones, usos sociales, culturales y políticos que tiene), referidos a por qué se lee, para qué se lee, cómo la lectura ayuda a construir una imagen o presencia social de los sujetos » Aplicar esta categoría a la enseñanza es un modo alternativo de enseñar modos eficaces de leer. En la dimensión sociocultural de la lectura se incorporan miradas desde varios territorios epistemológicos como la psicología social, la animación cultural, la antropología social, etc.

Sin pretender agotar el tema en este campo, son representativos los trabajos de Petit (1999), Gauthier y Graves (1995); Shera (1990); Chartier (1992, 1996), Cassany (2006), Ames (2002), Bernhart (2003), Casalmiglia (1997), Fairclough (1992, 1995) que representan una muestra del asedio al problema desde distintos ámbitos. Al mostrar que esta actividad humana es socializada e histórica la descarto como una mera actividad de descodificación, aunque ésta se realice en aquélla, porque la acción de descifrar un código establece solamente relaciones referenciales y biunívocas entre el signo y el referente. En el acápite del artículo la cita de Goethe señala la idea de práctica en construcción, de proceso permanente e inacabado de la praxis de la lectura.

Barthes (1975) concibe la lectura como «la hemorragia permanente», un disparador de asociaciones y de construcción subjetiva de significados. La asociación consagra el derecho al sentido amplio y múltiple. Resulta, por eso, una ruptura de estructuras y la entrada al territorio del deseo.

Certeau (1996) la define con la metáfora de la cacería furtiva. Allí establece los límites porque connota dos aspectos, por un lado el riesgo, lo prohibido, lo furtivo, y por otro la caza, actividad ancestral, juego de astucia que nos lleva al nomadismo, al viaje permanente, a la búsqueda permanente. Concuerda en que es una actividad de producción de sentido y consumo. En este aspecto se relaciona con lo que propone Álvarez Zapata.

Si la lectura es una actividad sociocultural contextualizada a la historia y por lo tanto marcada con fuerza por la temporalidad, su enseñanza también necesita contemplar y registrar esas mismas marcas. Desde la concepción sociocultural de la lectura el lector es intérprete y creador de significado, por eso se considera que el sujeto es un analfabeto funcional (iletrado) cuando el conocimiento sobre el lenguaje escrito está en la etapa del descifrado, no puede obtener del texto una interpretación ni llegar a producir escritura sobre el mismo.

Al aceptar la lectura como una acción sociocultural mediada por el contexto abrimos la posibilidad de considerar que existen distintos modos de leer de acuerdo a la intencionalidad de comunicación del texto y también relacionados con el contexto del lector.

\section{6. ¿Cómo leer?}

«Los lectores se multiplicaron, los textos escritos se diversificaron, aparecieron nuevos modos de leer y nuevos modos de escribir. Los verbos 'leer" y "escribir' habían dejado de tener una definición inmutable: no designaban (y tampoco designan hoy día) actividades homogéneas. Leer y escribir son construcciones sociales. Cada época y cada circunstancia histórica da nuevos sentidos a esos verbos». Ferreiro (2000).

¿Cuántos modos de leer existen? En la escuela la lectura es propuesta como una actividad única en una situación desnaturalizada, la del aula.

Dice Bombini (2008:24): «En investigaciones recientes referidas a los discursos acerca de la lectura en la Argentina producidos desde la escuela o más allá de ella en la última década 
(Bombini, 2002 y Cuesta, 2003), se observaba en éstos la fuerte presencia de referencias que hacen hincapié en el déficit, que describen las carencias de los sujetos y la ineficacia del sistema educativo, las falencias de los docentes y la pauperización de la cultura letrada».

El problema que se plantea en este punto es que la imposición de un modo unívoco de lectura es también la imposición que se realiza de forma asimétrica de los parámetros de una cultura o subcultura sobre otra. Esto nos lleva a la discusión acerca de los parámetros de evaluación acerca del cómo leer y para qué leer. Hay que preguntarse si los parámetros que determinan las metas de comprensión lectora tienen en cuenta las variaciones tanto de los aspectos socioculturales como de los diversos niveles de lenguaje de grupos sociales diferentes.

Aspirar a formar sujetos lectores debe incluir la posibilidad de que esos sujetos no se sientan excluidos de la cultura letrada y escolar. Es decir, tener en cuenta la tensión que toda operación homogeneizante, como es la escuela, impone.

Una posibilidad a tener en cuenta es tomar posición desde una mirada sociocultural y revisar, desde el punto de vista cualitativo, experiencias y situaciones donde la lectura sea puesta en valor desde la concepción y la representación de los sujetos que aprenden.

Es en ese punto donde pierde sentido el aspecto reduccionista de la comprensión lectora, porque en realidad la operación que pone en valor la lectura trasciende ampliamente esa categoría y la lectura se pone en situación de apropiación del lector, de allí la categoría planteada por Álvarez Zapata: MOCT (modo de producción y consumo textual).

En el mismo sentido va el trabajo de Rockwell (2001) que toma la idea de «apropiación» manejada por Chartier. Esta apropiación se plantea en referencia a lo que en el campo del lenguaje se denomina connotación, es decir, la multiplicidad de interpretaciones que ocurren en la recepción de un texto, las que están en el repertorio posible del lector y entre las que selecciona. Según señala Rockwell, la apropiación de un texto tiene límites, hasta allí se extiende la categoría de la comprensión lectora, pero luego aparece la creatividad y la construcción del lector que se apropia del texto y lo re-crea desde su momento histórico y su experiencia personal.

Estas prácticas proponen, de manera inevitable, una revisión del «aparato interpretativo escolar», como señala Bombini (ut supra) una nueva retórica y una nueva poética de los lectores que trascienden el ámbito de la lectura escolar y construyen lectores activos y protagonistas.

Al proponer esta forma de lectura sociocultural, comprensiva en lugar de exclusiva, se abre una posibilidad nueva según Bombini (ut supra): «Una nueva perspectiva sobre los lectores y sus modos de leer, una revisión de los presupuestos teóricos en relación con la lectura, el relato de experiencias interesantes -pero no necesariamente exitosas- desde las problematizaciones que presentan un abordaje metodológico específico que permite recuperar la riqueza de la lectura como empírea a ser leída e interpretada, que da voz al punto de vista de los lectores sobre sus propias experiencias y que invita a una producción escrita referida a la lectura misma, son algunos de los aportes de esta productiva línea de trabajo que aún necesita mayor desarrollo». Por esto las estrategias metodológicas de la lectura crítica son parte de una búsqueda cualitativa que ponga en valor los aspectos que definan una lógica contextual de la práctica de la lectura con la construcción de la comprensión y la interpretación desde una situación históricamente situada.

Frente a esta realidad y a esta propuesta de práctica situada para la enseñanza de la lectura surgen preguntas ineludibles:

¿Cuál es la teoría apropiada? ¿cómo realizar el recorte apropiado a la teoría? ¿cómo usarla en la práctica pedagógica? 
Ya situados en la práctica pedagógica estas cuestiones plantean un desafío metodológico para aplicar la teoría sociocultural de la lectura. Este desafío necesita contar con la narración de experiencias reales en las que la práctica productiva debe apoyarse con el objetivo de recuperar las acciones significativas y los distintos modos en que los sujetos se relacionan con la cultura letrada.

La lectura es propuesta en la escuela de hoy como instrumento de aprendizaje, como práctica funcional para la comunicación e información y como espacio lúdico de fruición. Desde esta polifonía modal será difícil que convoque a realizar alguna de estas actividades sino que las confundirá a todas, ya que cada una de ellas requiere de distintos modos de «leer».

Por lo tanto, para evitar esta confusión, en la práctica pedagógica es necesario establecer una tipología de la lectura y atenerse a ella al enseñar, ya que el modo de producción y el contexto son los que marcan la creación de sentido de un texto.

A modo de ejemplo, para situar una lectura desde el punto de vista sociocultural, veamos el siguiente texto: «El futbolista formado en Fénix partió del extremo derecho de la línea de cuatro volantes y resultó sospechoso de poseer un tercer pulmón» (Rodríguez, 2013. Periódico La Diaria. 14.2.13 Montevideo).

El texto pertenece a una crónica deportiva, lo que puede deducirse de lo escrito, pero lo demás depende de saber a qué partido se refiere, conocer la trayectoria de los futbolistas, quiénes fueron formados en Fénix que es un equipo de fútbol de Uruguay, qué posibles formaciones existen en el fútbol. Una persona que no se interese por el fútbol o que viva en un país lejano y no conozca los equipos uruguayos, por excelente lector que fuera perdería puntos si se le pidiera un test de comprensión lectora, salvo que se le orientara sobre el contexto con información extra.

En otro plano de lectura o en otro modo de producción textual, ¿cuántas personas pueden comprender en una primera lectura un escrito jurídico?

Hoy en día la tipología de textos es muy extensa. Desde textos corrientes como un correo electrónico o un instructivo para llenar un formulario hasta los datos de componentes y nutrición de los alimentos en sus envases; desde las «ayudas» de los programas y aplicaciones de la informática hasta textos con contenido científico, todos forman parte de la lectura diaria de las personas.

Frente a esta situación de la lectura, la contextualización y las prácticas deben ser revisadas y cuestionadas ya que los sujetos proceden de un ámbito sociocultural específico que les da herramientas de interpretación, pero la situación de la escuela y la enseñanza presentan un escenario artificial desnaturalizado que, a veces, puede entorpecer la lectura y los aprendizajes y confundir las evaluaciones.

Una docente de español como lengua materna me explicaba que sus alumnos de un curso básico de Secundaria no habían podido reconocer un texto periodístico como tal en una evaluación en clase. Le pregunté cuáles eran las marcas que ella estimaba que sus alumnos debían reconocer y me contestó con una enumeración de nombres técnicos del armado editorial de un periódico (título, copete, etc.). Luego hablamos de en qué soporte material debían reconocer esas características y resultó que la propuesta había sido hecha sobre un artículo fotocopiado del periódico. Esos alumnos no tuvieron la marca más notoria y más reconocible desde el contexto sociocultural, que señalaba el texto como un artículo periodístico, el soporte y la estructura y el tipo de papel que determinan que identifiquemos un objeto como un periódico, 
y se les pedía que reconocieran aspectos y nombres técnicos que la mayoría de las personas no manejan porque son específicos de determinadas prácticas y de determinada profesión.

La lectura es siempre una práctica social y situada, por lo tanto su enseñanza debería contemplar esta situación y establecer modos y tipos de lectura y acompañar a los lectores noveles y avanzados a construir prácticas cada vez más eficientes de lectura.

Al parecer es hora de revisar nuestras prácticas para alcanzar la meta de construir lectores hábiles, críticos y que disfruten de una herramienta que es productora de equidad y creadora de subjetividad y autoestima.

\section{Bibliografía}

Álvarez Zapata (2002). Del modo de leer como modo de producción y consumo textual. Revista Educación y Pedagogía. Medellín: Universidad de Antioquia Vol. XIV, No. 32, (enero - abril) 2002. Consultado el 6 de julio de 2013. http://aprendeenlinea.udea.edu.co/revistas/index.php/ revistaeyp/article/view/6739/6172

Ames (2002). Para ser iguales, para ser distintos. Consultado el 15 de febrero de 2013) http:// archivo.iep.pe/textos/DDT/paraser.pdf

Barthes, R. (1994). El susurro del lenguaje. Buenos Aires. Paidós.

Bernhart et al. (2003). La enseñanza de la lectura. Consultado el 15 de febrero de 2013 http://www.ibe.unesco.org/fileadmin/user_upload/Publications/Educational_Practices/ EdPractices_12s.pdf

Bombini, G. (2002). Sabemos poco acerca de la lectura. en Lenguas Vivas. Publicación del Instituto de Enseñanza Superior "Juan Ramón Fernández". Año 2, № 2, octubre-noviembre, Buenos Aires.

Bombini, G. (2004). Los arrabales de la literatura. La historia de la enseñanza de la literatura en la escuela secundaria de la Argentina (1870-1960)" Tesis de doctorado. Buenos Aires. Miño y Dávila y Universidad de Buenos Aires (UBA). Facultad de Filosofía y Letras.

Casalmiglia, H. (1997) (coord.). UPF, Actas del I Simposio Internacional de Análisis del Discurso. UC. 20/22-4-98 Consultado el 15 de febrero de 2013. http://www.upf.edu/pdi/dtf/ daniel_cassany/analies2.htm

Cassany, D. (2006). Tras las líneas. Barcelona: Anagrama.

Certeau, M. (1996). La invención de lo cotidiano. I.UI, México, Frag. Capítulo VII

Consultado el 15 de febrero de 2013 http://popularymasiva.files.wordpress.com/2010/03/ andares_de_la_ciudad.pdf

Chartier (1992, 1996). El mundo como representación. Historia cultural. Barcelona. Gedisa.

Chartier, A. M. y Hébrard, J. (2002) La lectura de un siglo a otro. Discursos sobre la lectura» Barcelona. Gedisa.

Eckermann, J. P. (2008). Conversaciones con Goethe U.N.A.M.

Fairclough (1992, 1995). General introduction. En Critical discourse analysis. The critical study of language. London and New York: Longman.

Ferreiro, E. (2000). Leer y escribir en un mundo cambiante. Consultado el 15 de febrero de 2013. http://www.foro-latino.org/.

Gauthier y Graves (1995). Lectura y sociedad. Hojas de Lectura. Bogotá. No. 35, pp. 6-13. 
Goodman, Y. (1992). Infancia y aprendizaje Journal for the Study of Education and Development, № 58, 1992, Consultado el 15 de febrero de 2013 http://dialnet.unirioja.es/ servlet/articulo?codigo $=48394$

Goswami y Bryant (1990). Phonological awareness and learning to read a first language. Consultado el 15 de febrero de 2013. http://lotos.library.uu.nl/publish/articles/000170/bookpart. pdf

Mendoza Fillola (1991). Intertexto lector. Consultado el 15 de febrero de 2013. http://books. google.com.uy/books?hl=es\&lr=\&id=ilhlZqMnqdAC\&oi=fnd\&pg=PA23\&dq=Mendoza+Fillola $++(1991 \&$ ots=_9vneyEMOM\&sig=t2VrAMZvIn1s0TIGYOr9GSL9_8k\#v=onepage\&q=Mendo za\%20Fillola\%20\%20(1991\&f=false

Molina García, S. (1991). Psicopedagogía de la lectura. CEPE. Madrid.

Petit, M. (1999). Nuevos acercamientos a los jóvenes y la lectura. México: FCE.

ProLEE, (2011). Consultado el 15 de febrero de 2013 http://www.uruguayeduca.edu.uy/ repositorio/prolee/index.html

Rockwell, E. (2005). La lectura como práctica cultural: conceptos para el estudio de los libros escolares. en Lulú Coquette. Revista de Didáctica de la Lengua y la Literatura, año 3. № 3. Buenos Aires. El Hacedor. Jorge Baudino.

Shera, J. Los fundamentos de la educación bibliotecológica. México: UNAM, CUIB.

Stanovich y Stanovich, (1999). Using research and reason in education. W, D. C.: USDE.

Sylva (2001). "Effective Provision of Pre-school Education". Consultado el 15 de febrero de 2013. http://eppe.ioe.ac.uk/eppe/eppepdfs/beramanchester04.pdf

Weinberger, (1996) cit. en Brooks, (2000) British Educational Research Journal. Volume 26, Issue 1, 2000. Consultado el 15 de febrero de 2013 http://www.tandfonline.com/doi/ pdf/10.1080/014119200109543 15.2.13.23:00

Fecha de recibido: 15/02/2013

Fecha de aceptación: 05/06/2013

* Máster en Educación, Universidad ORT Uruguay. Subdirectora, Liceo Nº 3 de Montevideo. Profesora de Literatura, Instituto de Profesores Artigas. Integrante del equipo del Proyecto PROLee del Consejo Directivo Central de la Administración Nacional de Educación Pública. Miembro del Grupo para la Inclusión y Calidad Educativas a través de Ocuparnos de la Lectura y Escritura en todas las Materias. 